\title{
CXCL12/CXCR4 axis regulates neovascularization and lymphangiogenesis in sutured corneas in mice
}

\author{
LING-LING DU and PING LIU
}

\author{
Department of Ophthalmology, The First Affiliated Hospital of Harbin Medical University, \\ Harbin, Heilongjiang 150001, P.R. China
}

Received July 4, 2015; Accepted March 2, 2016

DOI: $10.3892 / \mathrm{mmr} .2016 .5179$

\begin{abstract}
The present study aimed to determine the plausible functional role of chemokine (C-X-C motif) ligand 12 (CXCL12)/chemokine (C-X-C motif) receptor 4 (CXCR4) in inflammatory corneal hemangiogenesis and lymphangiogenesis in vivo. Corneal hemangiogenesis and lymphangiogenesis were induced by placing an 11-0 nylon suture in an intrastromal position. The expression levels of the vascular endothelial growth factor (VEGF) family, CXCL12 and CXCR4 in the corneas were investigated in the corneas using reverse transcription-quantitative polymerase chain reaction and immunohistochemistry. Corneal hemangiogenic and lymphangiogenic responses were assessed by immunofluorescence using specific antibodies against cluster of differentiation 31 and lymphatic vessel endothelial hyaluronan receptor-1. Subconjunctival injection of AMD3100 to the sutured corneas was also performed. CXCL12/CXCR4 mRNA and protein expression levels increased markedly in suture-induced corneal neovascularization (CNV) and decreased with AMD3100 treatment. Hemangiogenesis and lymphangiogenesis were captured in images using immunofluorescence and were shown to be markedly increased with suture placement and reduced with AMD3100 treatment. VEGF-A/VEGFR-1 and VEGF-C/VEGFR-3 mRNA expression levels were upregulated in the suture placement and control groups, whereas the expression levels of all the factors were downregulated in the AMD3100 treatment group. The results from the present study demonstrated that CXCL12/CXCR4 interactions regulate hemangiogenesis and lymphangiogenesis in suture-induced CNV. AMD3100 may be a novel therapeutic target for the prevention of blindness.
\end{abstract}

Correspondence to: Dr Ping Liu, Department of Ophthalmology, The First Affiliated Hospital of Harbin Medical University, 23 Youzheng Street, Nangang, Harbin, Heilongjiang 150001, P.R. China

E-mail:ping_liup@163.com

Key words: CXCL12/CXCR4, corneal inflammation, hemangiogenesis, lymphangiogenesis

\section{Introduction}

The maintenance of corneal transparency is required for optimal vision, thus, the cornea must preserve avascularity and alymphaticity (1). It is widely recognized that new blood and lymphatic vessels in the cornea are strong risk factors for corneal transparency. Corneal neovascularization (CNV), also referred to as angiogenesis or hemangiogenesis (HA), is the formation of ectopic corneal vasculature from preexisting vasculature (2). $\mathrm{CNV}$ is promoted by a wide variety of proangiogenic factors, including vascular endothelial growth factor (VEGF), basic fibroblast growth factor (bFGF), interleukin-6, platelet-derived growth factor (PDGF), hepatocyte growth factor (3-7). In addition, recent studies have demonstrated that anti-angiogenic factors, such as angiostatin and endostatin, inhibit CNV (3).

Lymphangiogenesis ( $\mathrm{LG}$ ) is the formation of novel lymphatic vessels from pre-existing vasculature. In contrast to angiogenesis, research on the lymphatic system has been slowed by a lack of molecular markers to identify the vessels. The recent discovery of several lymphatic endothelial molecular markers, including VEGF receptor-3 (VEGFR-3), lymphatic vessel endothelial hyaluronan receptor-1 (LYVE-1), and Prox-1, has enabled further research and progress in the field (8-10).

Signaling via VEGF-C/D and VEGFR3 is a central pathway for LG $(11,12)$. VEGF- $\mathrm{C}^{+-}$mice are viable but suffer from lymphatic deficiency and subsequent lymphedema (13). Similarly, inhibition of VEGFR3 signaling during the formation of lymphatic vessels induces lymphatic regression and lymphedema in mouse embryos and neonates (14). VEGF-A is widely used for angiogenesis research as it promotes several processes of angiogenesis, including proteolytic activities (dissolution of the membrane of the original vessel), endothelial cell proliferation, migration, and capillary tube formation. Notably, the requirement of VEGF in corneal angiogenesis was demonstrated by the inhibition of $\mathrm{NV}$ following stromal implantation of an anti-VEGF blocking antibody in a rat model (15).

Chemokine (C-X-C motif) ligand 12 (CXCL12) is a member of the CXC subfamily of chemokine peptides, also known as stromal cell-derived factor-1 (SDF-1). However, unlike other chemokines that interact with multiple G-protein coupled receptors, CXCL12 mediates its effects via its only known receptor, chemokine (C-X-C motif) receptor 4 (CXCR4). CXCL12 is highly conserved with $99 \%$ homology between humans and mice, allowing it to exert effects across species barriers (16). 
The CXCL12/CXCR4 axis is essential for multiple biological processes, including development, hematopoiesis, organogenesis, and vascularization (17-20). Furthermore, CXCR4 is highly expressed in a variety of cancers (21). The interaction between CXCL12 and CXCR4 is important in tumorigenesis (22) and metastasis $(21,23)$. AMD3100, a well-known antagonist of CXCR4, reversibly inhibits interaction of CXCL12 and CXCR4 (24). Previous studies have identified the CXCL12/CXCR4 axis is associated with NV (25-27). However, the association between the CXCL12/CXCR4 axis and LG remains to be elucidated.

The role of the CXCL12 (SDF-1)/CXCR4 axis in mediating $\mathrm{LG}$ in suture-induced $\mathrm{CNV}$ remains to elucidated. To investigate the roles of CXCR4 signaling, the process of suture-induced CNV was analyzed using mice with suture placement or CXCR4 antagonist (AMD3100)-treated mice in comparison with control-treated mice. The present study also investigated whether VEGF signaling was involved in the molecular signaling pathway resulting in corneal LG due to placement of a suture.

\section{Materials and methods}

Animals and anesthesia. All animal protocols were approved by the local animal care committee of The First Affiliated Hospital of Harbin Medical University (Harbin, China), and were in accordance with the Association for Research in Vision and Ophthalmology Statement for the Use of Animals in Ophthalmic and Vision Research. A total of 25 male C57BL/6 mice (age, 6-8 weeks) were purchased from the Changchun Animal Center (Changchun, China). Mice were housed in three groups (9 mice in control group, and 8 mice in AMD3100 and suture placement groups each) under a 12-h light/dark cycle at moderate temperature and humidity with free access to food and water. Animals were anesthetized with a mixture of ketamine and xylazine (Zhejiang Jiuxu Pharmaceutical Co., Ltd.; Jinhua, China) at $120 \mathrm{mg} / \mathrm{kg}$ body weight and $20 \mathrm{mg} / \mathrm{kg}$ body weight, respectively). At the end of the experiment, the mice were sacrificed by $\mathrm{CO}_{2}$ inhalation.

Suture-induced CNV. The mouse model of suture-induced inflammatory CNV was used as previously described (28). The central cornea was marked with a $2-\mathrm{mm}$ diameter trephine and three 11-0 nylon sutures (Lingqiao, Ningbo, China) were placed in the intrastromal position. The outer point of suture placement was selected to be near the limbus, and the inner point was selected to be near the corneal center equidistant from the limbus. Sutures were removed after 7 days. Mice were randomly selected to receive subconjunctival injection of the CXCR4 antagonist, AMD3100 (1 g/l; Abcam, Cambridge, UK) or balanced salt solution once a day from day 1 of the suture-induced model.

Measure of suture-induced CNV. CNV was examined using a slit lamp every other day following the corneal suture. Measurements of NV were made using a slit lamp by an ophthalmologist. Vessel growth onto the cornea was recorded in millimeters on day 7. CNV was quantified by calculating the wedge-shaped area of vessel growth with the formula: $\mathrm{A}=\mathrm{C} / 12 \times 3.1416\left[\mathrm{r}^{2}-(\mathrm{r}-1)^{2}\right]$, where $\mathrm{A}$ is the area, $\mathrm{C}$ is the time
Table I. Primers used in the reverse transcription-quantitative polymerase chain reaction.

\begin{tabular}{|c|c|}
\hline Gene & Sequence $\left(5^{\prime}-3^{\prime}\right)$ \\
\hline \multirow[t]{2}{*}{ GAPDH } & F: GTATTGGGCGCCTGGTCACC \\
\hline & R: CGCTCCTGGAAGATGGTGATGG \\
\hline \multirow[t]{2}{*}{ VEGF-A } & F: ACACGGTGGTGGAAGAAGAG \\
\hline & R: CAAGTCTCCTGGGGACAGAA \\
\hline \multirow[t]{2}{*}{ VEGF-C } & F: CTACAGATGTGGGGGTTGCT \\
\hline & R: GATTGGCAAAACTGATTGTGAC \\
\hline \multirow[t]{2}{*}{ VEGFR-1 } & F: CTGGACTGAGACCAAGCCCAAG \\
\hline & R: GCTCAGATTCATCGTCCTGCAC \\
\hline \multirow[t]{2}{*}{ VEGFR-3 } & F: CTCTGACCTAGTGGAGATCCTG \\
\hline & R: CTTCGGTGATATGTAGAGCTGTG \\
\hline \multirow[t]{2}{*}{ CXCR4 } & F: AGCATGACGGACAAGTACC \\
\hline & R: GATGATATGGACAGCCTTACAC \\
\hline \multirow[t]{2}{*}{ CXCL12 } & F: GAGAGCCACATCGCCAGAG \\
\hline & R: TTTCGGGTCAATGCACACTTG \\
\hline
\end{tabular}

F, forward; R, reverse; VEGF, vascular endothelial growth factor; VEGFR, VEGF receptor; GAPDH, glyceraldehyde-3-phosphate dehydrogenase; CXCR4, chemokine (C-X-C motif) receptor 4; CXCL12, chemokine (C-X-C motif) ligand 12.

(h), 1 is the radius from the center to the border of vessel growth, and $r$ is the radius of the cornea (29).

Immunohistochemistry. Corneas were cut and fixed in $10 \%$ neutral buffered formalin (Beijing Yili Fine Chemicals Co., Ltd.; Beijing, China) for $24 \mathrm{~h}$. Paraffin-embedded tissue sections $(4 \mu \mathrm{m})$ were deparaffinized, rehydrated, and treated with $0.3 \%$ hydrogen peroxide in methanol (Beyotime Institute of Biotechnology, Inc., Haimen, China) for $30 \mathrm{~min}$, to eliminate endogenous peroxidase activity. The tissue sections were incubated for $60 \mathrm{~min}$ at room temperature with rabbit anti-mouse CXCR4 polyclonal antibody (1:200 dilution; cat. no. ab2074; Abcam). Following three washes of 3 min with phosphate-buffered saline (PBS; Beyotime Institute of Biotechnology, Inc.), a 3,3'-diaminobenzidine detection kit (PV9000; ZSGB-BIO, Beijing, China) was used for CXCR4 staining, according to the manufacturer's protocols. Images were captured with the Leica DM4000B biological microscope equipped with a Leica DFC 550 digital camera and Leica Application Suite version 4.2.0 software (Leica Microsystems GmbH, Wetzlar, Germany).

RNA isolation and reverse transcription-quantitative polymerase chain reaction ( $R T-q P C R)$. Total RNA was extracted using TRIzol reagent (Invitrogen; Thermo Fisher Scientific, Inc., Waltham, MA, USA) according to the manufacturer's protocols. Total RNA (400 ng) was reverse transcribed using the PrimeScript ${ }^{\mathrm{TM}}$ RT reagent kit with gDNA Eraser (Takara Biotechnology Co., Ltd., Dalian, China). qPCR was performed using SYBR ${ }^{\circledR}$ Premix Ex Taq $^{\mathrm{TM}}$ II (Takara Biotechnology Co., Ltd.) in a LightCycler 480 Real-time PCR system (Roche Diagnostics, Basel, Switzerland). The thermocycling conditions were as follows: Initial denaturation step of $95^{\circ} \mathrm{C}$ for $30 \mathrm{sec}$; 40 cycles of $95^{\circ} \mathrm{C}$ for $5 \mathrm{sec}$ and $60^{\circ} \mathrm{C}$ for $30 \mathrm{sec}$; followed by 


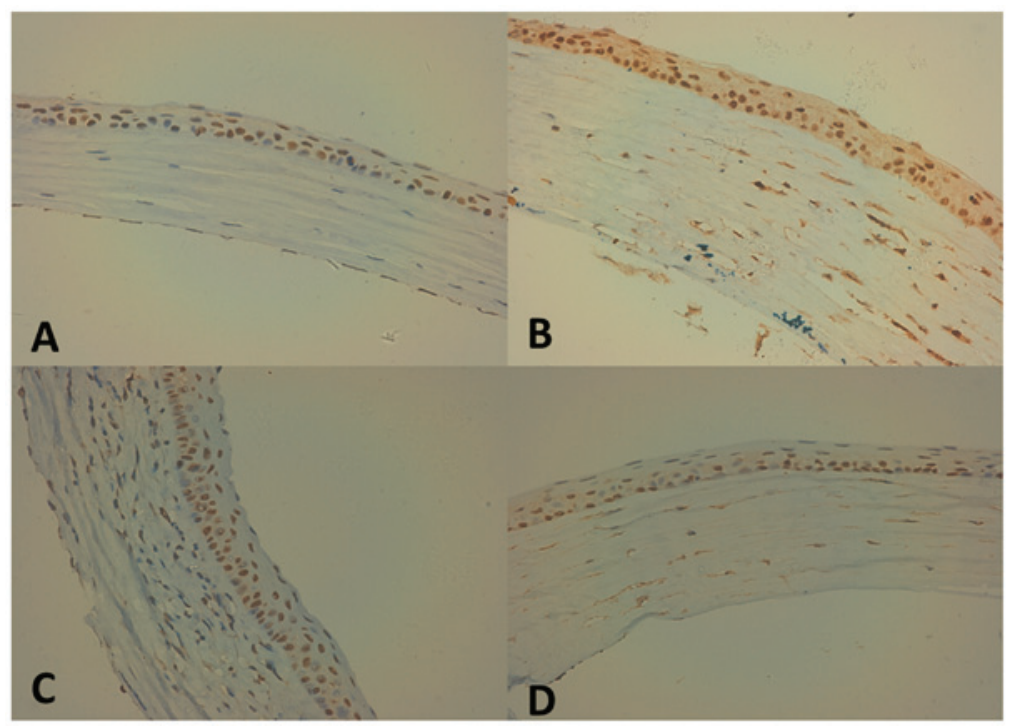

Figure 1. CXCR4 expression levels in the cornea were analyzed using immunohistochemical staining. (A) CXCR4 was detected in epithelial cells in normal corneas (B) CXCR4 expression levels were markedly upregulated on day 7 following corneal suture placement. (C) High expression levels of CXCR4 were observed in the cornea on day 7 following balanced salt solution subconjunctival injection (control group). (D) Weak expression levels of CXCR4 were observed following AMD3100 subconjunctival injection. The yellow areas demonstrate the expression of CXCR4. Magnification, $\mathrm{x} 400$. Scale bar, $100 \mu \mathrm{m}$. CXCR4, chemokine (C-X-C motif) receptor 4.

an additional denaturation step of $95^{\circ} \mathrm{C}$ for $5 \mathrm{sec}$ and $60^{\circ} \mathrm{C}$ for $60 \mathrm{sec}$, as a subsequent melt curve analysis to check amplification specificity. The assays were conducted three times, and were performed in triplicate. Results were derived from the $2^{-\Delta \Delta \mathrm{Cq}}$ method (30) and glyceraldehyde-3-phosphate dehydrogenase served as an internal control for normalization. Primers used in the RT-qPCR are presented in Table I.

Western blot analysis. The corneas were harvested and lysed in ice-cold radioimmunoprecipitation lysis buffer (Beyotime Institute of Biotechnology, Inc.) with the addition of protease inhibitors. Following separation by $10 \%$ sodium dodecyl sulfate-polyacrylamide gel electrophoresis at $120 \mathrm{~V}$ for $2 \mathrm{~h}$, proteins were transferred onto nitrocellulose membranes (Pall Life Science, Port Washington, NY, USA). The membranes were incubated in blocking solution of $2 \%$ bovine serum albumin (BSA; Beyotime Institute of Biotechnology, Inc.) in Tris-buffered saline with Tween-20 (Beyotime Institute of Biotechnology, Inc.) for $1 \mathrm{~h}$ at room temperature, then incubated with rabbit anti-mouse CXCR4 polyclonal antibody and rabbit anti-mouse CXCL12 polyclonal antibody (cat. no. ab25117; Abcam) at 1:1,000 dilution overnight at $4^{\circ} \mathrm{C}$. Each step was followed by extensive washing. $\beta$-actin was used as loading control (mouse anti-mouse $\beta$-actin monoclonal antibody; cat. no. A00702-100; 1:1,000 dilution; GenScript, Nanjing, China). The membranes were then incubated with horseradish peroxidase-conjugated rabbit anti-mouse polyclonal immunoglobulin (Ig)G (1:5,000 dilution; cat. no. A9044; Sigma-Aldrich, St. Louis, MO, USA) or goat anti-rabbit polyclonal IgG secondary antibody (cat. no. A0545; 1:5,000 dilution Sigma-Aldrich) for $1 \mathrm{~h}$ at room temperature and developed using an enhanced chemiluminescence system (GE Healthcare, Little Chalfont, UK). Images were captured on X-ray film.

Corneal immunofluorescence microscopy assays and quantification. The immunofluorescence experiments were performed as previously described (31). The excised corneas from the $\mathrm{CNV}$ assay were rinsed in PBS and fixed in acetone (Beyotime Institute of Biotechnology, Inc.) for $30 \mathrm{~min}$. Following washing and blocking with $2 \%$ BSA in PBS for $2 \mathrm{~h}$, the corneas were stained overnight at $4^{\circ} \mathrm{C}$, with a rabbit anti-mouse LYVE-1 antibody (cat. no. ab14917; 1:500 dilution; Abcam) and a rat anti-mouse cluster of differentiation (CD)31 antibody (1:100; cat. no. 550274; BD Pharmingen, San Diego, CA, USA). On day 2, the tissue was washed three times in PBS and stored at $4^{\circ} \mathrm{C}$ in the dark. LYVE-1 antibody was detected with an Alexa Fluor ${ }^{\circledR}$ 647-conjugated goat anti-rabbit IgG antibody (1:200; cat. no. A-21244; Invitrogen; Thermo Fisher Scientific, Inc.) and the CD31 antibody was detected with Alexa Fluor $488^{\circledR}$-conjugated goat anti-rat IgG polyclonal antibody (1:200; cat. no. A-11006 Invitrogen; Thermo Fisher Scientific, Inc.).

The stained whole mounts were analyzed with a fluorescence microscope (EVOS f1; Thermo Fisher Scientific, Inc.). Each whole mount picture was quantified using Image J software version 1.24o (National Institutes of Health, Bethesda, MD, USA) analysis software as described previously (32). The mean vascularized area of the suture placement was defined as being $100 \%$; vascularized areas were then relative to this value (vessel ratio).

Statistical analysis. Statistical analysis was performed by the Student's t-test using SPSS version 13.0 software (Armonk, NY, USA). Results were expressed as the mean \pm standard error of the mean and $\mathrm{P}<0.05$ was considered to indicate a statistically significant difference. Graphs were drawn using GraphPad Prism, version 5.0 software (GraphPad Software, Inc., La Jolla, CA, USA).

\section{Results}

Expression levels of CXCL12 and CXCR4 in a mouse model of suture-induced inflammatory $C N V$. The present study 
A

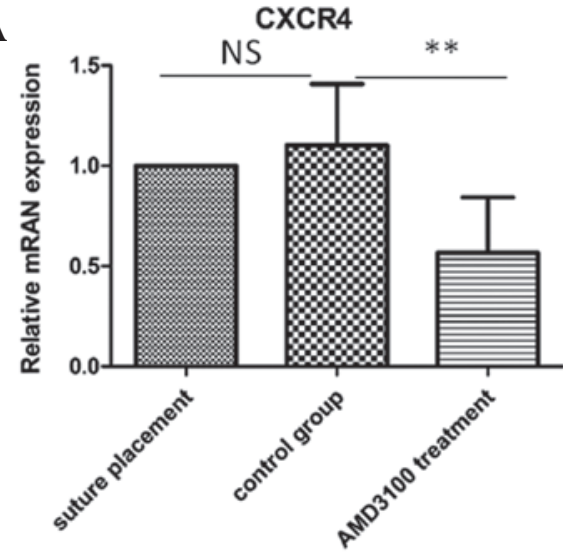

C

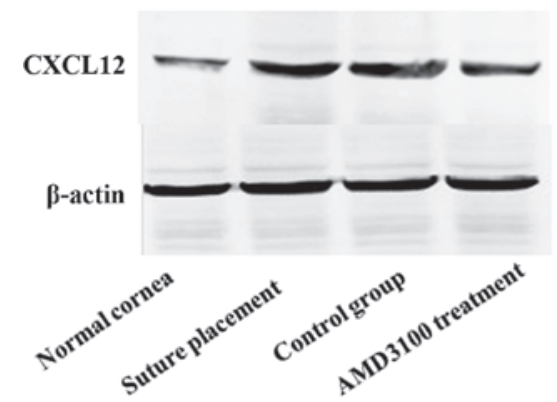

B
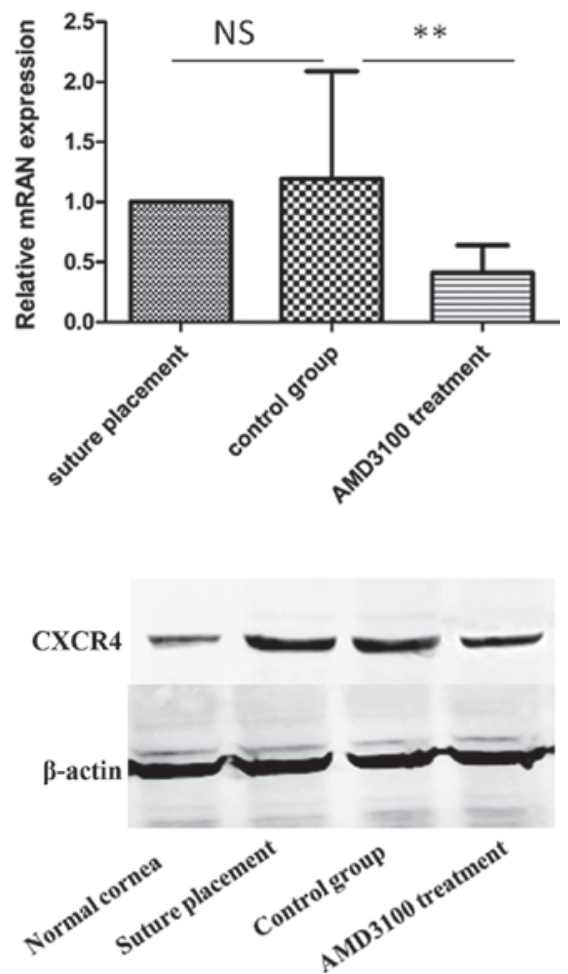

Figure 2. CXCL12/CXCR4 expression levels in normal and treated corneas. (A and B) Quantitative polymerase chain reaction analysis of CXCR4/CXCL12 mRNA was performed in corneas following suture placement and AMD3100 treatment. (C) Western blot assays demonstrated the protein expression levels of corneal CXCL12/CXCR4 under different conditions. The images are representative of three independent experiments. $\beta$-actin served as a loading control. ${ }^{* *} \mathrm{P}<0.01$. NS, not significant; CXCL12, chemokine (C-X-C motif) ligand 12; CXCR4, chemokine (C-X-C motif) receptor 4.
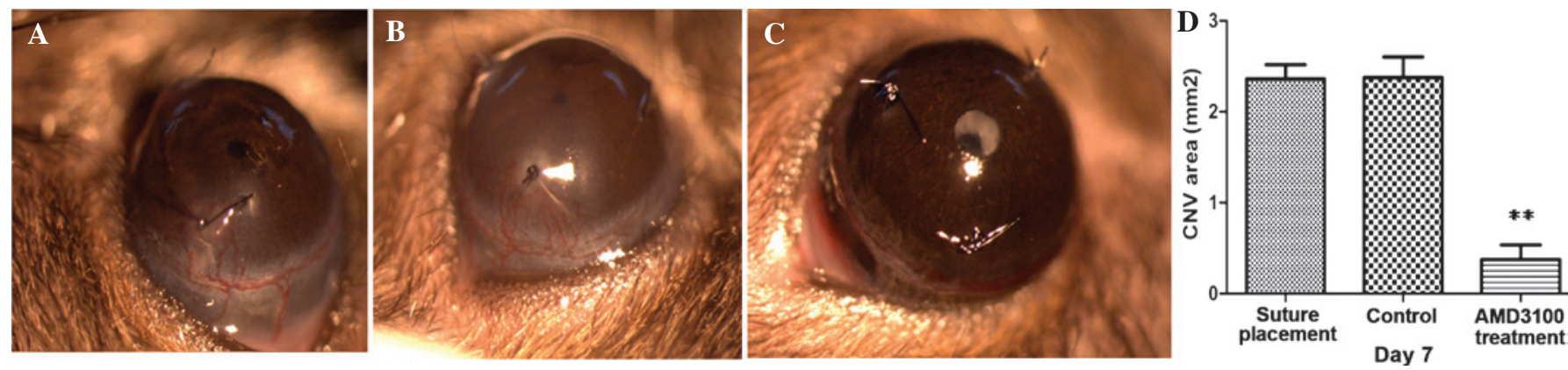

Figure 3. CNV was examined by slit lamp on day 7. (A) Suture-induced CNV (B) Suture-induced CNV with subconjunctival injection of balanced salt solution. (C) Suture-induced CNV with subconjunctival injection of AMD3100. CNV was measured and data are expressed as the mean + standard deviation (n=5). (D) The AMD3100-treated group demonstrated decreased CNV area. ${ }^{* *} \mathrm{P}<0.01$ vs. the suture-induced $\mathrm{CNV}$ and balanced salt solution subconjunctival injection groups. CNV, corneal neovascularization.

analyzed CXCL12 and CXCR4 mRNA and protein expression levels in normal and vascularized corneas by RT-qPCR, immunohistochemistry and western blot analysis. The immunohistochemical staining demonstrated CXCR4 was only detected in epithelial cells in normal corneas (Fig. 1A). Immunohistochemical staining showed a high expression of CXCR4 in corneas on day 7 following suture placement in the control group, and a weak expression of CXCR4 in AMD3100 subconjunctival injection (Fig. 1B-D). CXCL12 and CXCR4 mRNA was detected at low levels in normal eyes, but was significantly increased following suture placement and decreased with treatment with AMD3100 (P<0.01; Fig. 2A and B). CXCL12 and CXCR4 mRNA expression levels presented no significant change between the suture placement and control groups (P>0.05; Fig. 2A and B). Furthermore, the western blot assay also indicated a marked upregulation of CXCL12 and CXCR4 expression levels in the suture placement and control groups, and the downregulation of CXCL12 and CXCR4 expression levels with AMD3100 treatment (Fig. 2C). The increased mRNA and protein expression levels of CXCL12 and CXCR4 resulted in further investigation into whether CXCL12/CXCR4 interactions are involved in suture-induced CNV. Slit lamp examinations demonstrated that untreated corneas were avascular, however, suture placement markedly increased the vascular areas in corneas and the vascular areas decreased following AMD3100 treatment $(\mathrm{P}<0.01$; Fig. 3). Slit lamp 


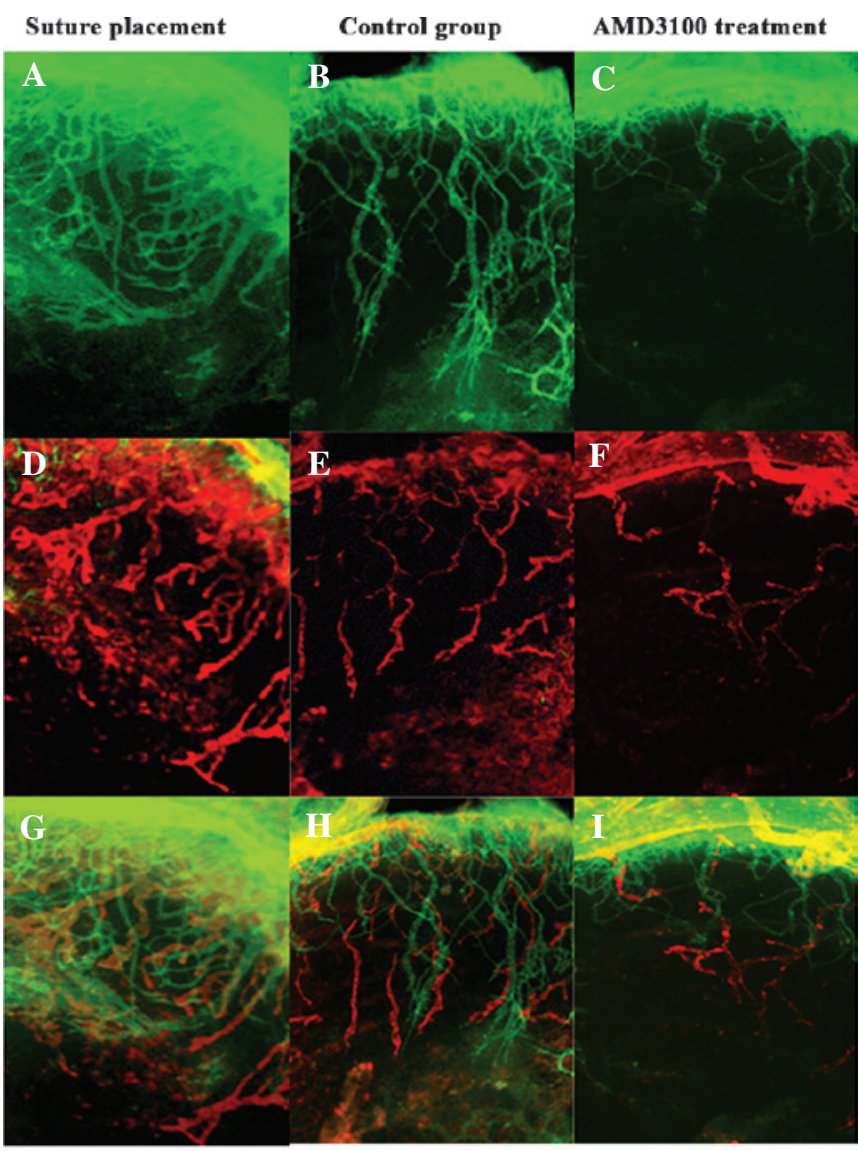

J

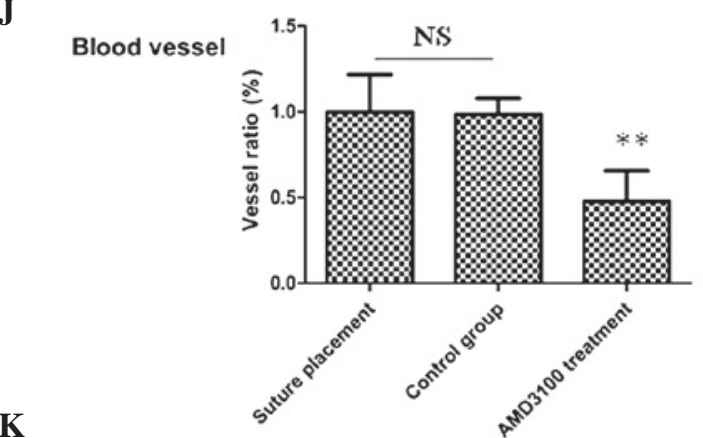

Lymphatic vesse

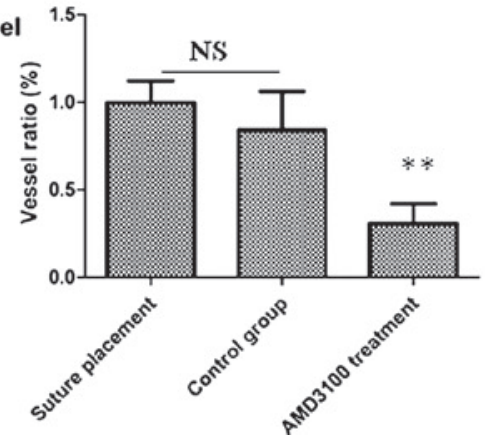

Figure 4. CXCL12/CXCR4 axis regulates CNV and LG using immunofluorescence. (A-C) Representative images showing that suture placement on day 7 (D-I) Representative segments of corneal whole-mounts (blood vessels stained green using CD31/Alexa Fluor ${ }^{\circledR} 488$ and lymphatic vessels stained red using LYVE-1/Alexa Fluor $\left.{ }^{\circledR} 647\right)$ in eyes treated with balanced salt solution $(\mathrm{B}, \mathrm{E}$ and $\mathrm{H})$ or AMD3100 (C, F and I) (magnification, x40). (J and K) Summarized data showing that areas of CNV and LG are not significantly different between the control and suture placement groups. The area of CNV following AMD3100 treatment was smaller than in the suture placement and control groups. (A, D and G) $\mathrm{n}=8$ mice, $(\mathrm{B}, \mathrm{E}$ and $\mathrm{H}) \mathrm{n}=9$ mice and $(\mathrm{C}, \mathrm{F}$ and $\mathrm{I}) \mathrm{n}=8$ mice. $^{* *} \mathrm{P}<0.01$ vs. suture placement and control groups. NS, not significant; CXCL12, chemokine (C-X-C motif) ligand 12; CXCR4, chemokine (C-X-C motif) receptor $4 \mathrm{CNV}$, corneal neovascularization; LG, lymphangiogenesis. examination was consistent with results from the analysis of mRNA and protein expression levels.

CXCL12/CXCR4 axis regulates CNV. CXCL12 is one of the major chemokines associated with $\mathrm{CXCR} 4$, thus, the present study hypothesized that the CXCL12/CXCR4 axis regulates $\mathrm{NV}$ and LG. To confirm the bioactivity of CXCL12/CXCR4 on NV, a mice model of suture-induced CNV was established in vivo. The effect of $\mathrm{CNV}$ was evaluated by comparing the $\mathrm{CNV}$ area between mice from the suture placement and AMD3100 treatment groups on day 7 (Fig. 3). The results demonstrated that the size of the areas of $\mathrm{CNV}$ indicate no significant difference between the control and the suture placement groups. The area following AMD3100 treatment was smaller than that in the suture placement and control groups, suggesting CXCL12/CXCR4 axis regulates $\mathrm{CNV}$ $(0.54 \pm 0.11,2.34 \pm 0.24$ and $2.65 \pm 0.32$, respectively; Fig. 3A-C). Corneal suture placement induced a robust $\mathrm{CNV}$ response that emerged from day 3 and reached suture placement sites at day 7 following suture injury (31). The densities of platelet endothelial cell adhesion molecule-1 (CD31)-positive blood vessels were detected by immunofluorescence on day 7 (Fig. 4A-C). Quantitative immunofluorescence and morphometric analyses demonstrated that the number of blood vessels in mice treated with AMD3100 was significantly decreased $(\mathrm{P}<0.01)$, whereas the number of blood vessels in the suture placement group showed no changes compared with balanced salt solution group ( $\mathrm{P}=0.46$; Fig. $4 \mathrm{~J})$. These results suggest that the CXCL12/CXCR4 axis is associated with CNV.

CXCL12/CXCR4 axis regulates $L G$. Lymphatic vessels express multiple specific markers, including LYVE-1, Prox-1, and VEGFR-3 (8-10). LYVE-1 is specifically expressed on newly formed lymphatic vessels but not on blood vessels in the mouse corneal model (33). Thus, the present study used LYVE-1 as a specific maker for detection of lymphatic vessels in the present study. On day 7, the densities of LYVE-1 positive lymphatic vessels were detected by immunofluorescence. The lymphatic vessels of suture placement had vascular 'tree-like' structures (Fig. 4D-F). Quantitative immunofluorescence and morphometric analyses demonstrated that corneal LG was induced by suture placement and the vessel ratio of lymphatic vessels in mice treated with AMD3100 were significantly decreased $(\mathrm{P}<0.01$; Fig. $4 \mathrm{~K})$ compared with the suture placement and control groups. The present study supports that CXCL12/CXCR4 regulates corneal LG.

CXCL12/CXCR4 pathway is dependent on the $V E G F-A / V E G F R-1$ pathway in the regulation of $C N V$. VEGF-A/VEGFR-1 is considered to induce angiogenesis. To investigate whether VEGF-A/VEGFR-1 was associated with CXCL12/CXCR4, RT-qPCR for VEGF-A/VEGFR-1 was performed. VEGF-A/VEGFR-1 mRNA expression levels were upregulated in the suture-induced CNV and control groups (Fig. 5), whereas VEGF-A/VEGFR-1 expression levels in the corneas treated with AMD3100 were downregulated. The results are consistent with the results of CXCL12/CXCR4. Thus, these results demonstrate that the CXCL12/CXCR4 pathway is dependent on the VEGF-A/VEGFR-1 pathway in regulating $\mathrm{CNV}$. 

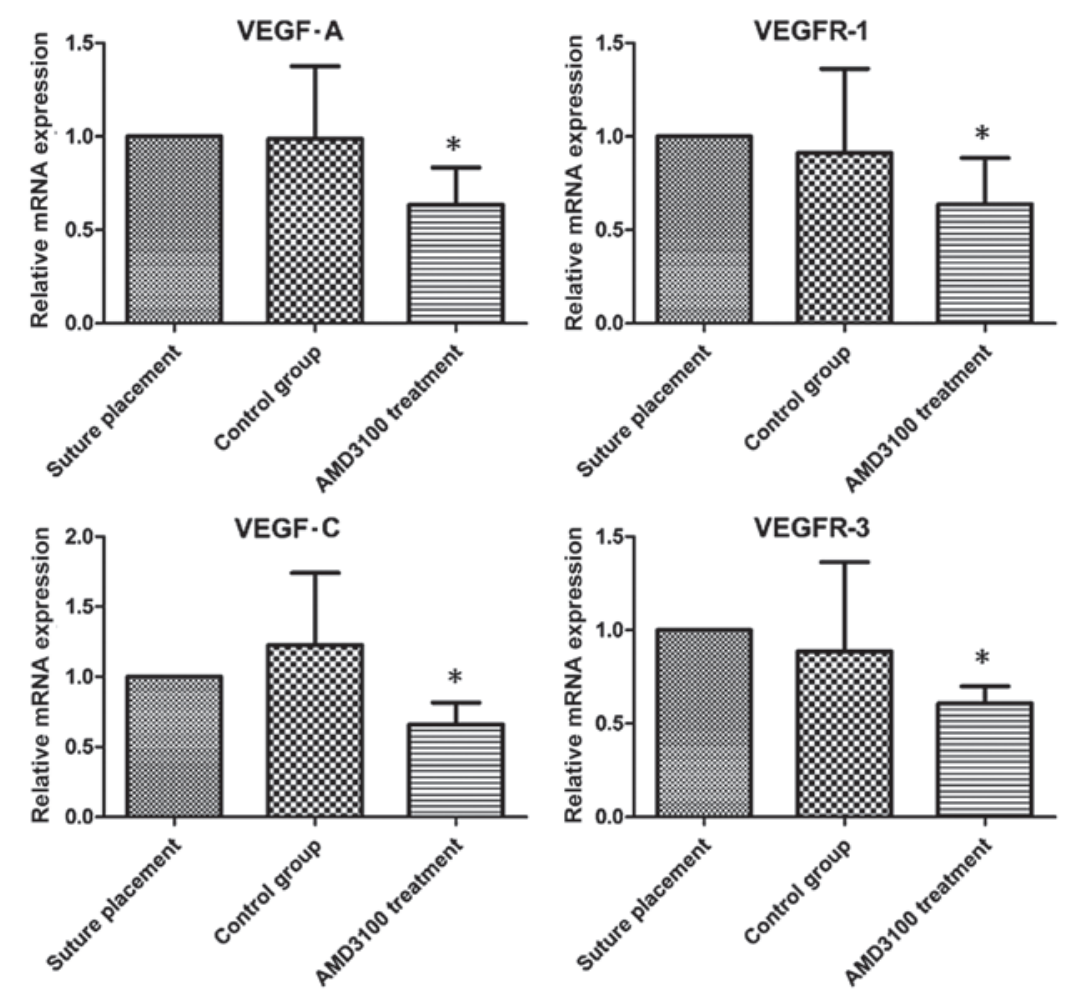

Figure 5. Representative graphs show the mRNA expression levels of VEGF-A, VEGF-C, VEGFR-1 and VEGFR-3 as measured by reverse transcription-polymerase chain reaction. VEGF-A, VEGF-C, VEGFR-1 and VEGFR-3 expression levels indicated no significant changes (all P $>0.05$ ) between suture placement and balanced salt solution subconjunctival injection groups, and the expression levels of all four factors was decreased to a large extent in AMD3100-treated mice. "P<0.05 vs. suture placement and control groups. VEGF, vascular endothelial growth factor; VEGFR, VEGF receptor.

CXCL12/CXCR4 pathway is dependent of the $V E G F-C / V E G F R-3$ pathway in the regulation of $L G$. The abovementioned results demonstrated that CXCL12/CXCR4 mediates LG. However, whether CXCL12/CXCR4 mediation of LG is associated with VEGF-C/VEGFR-3 remains to be elucidated. To further investigate the roles of VEGF-C and VEGFR-3 in suture-induced CNV, RT-qPCR was used to examine mRNA expression levels. VEGF-C and VEGFR-3 expression levels were significantly downregulated in mice treated with AMD3100 $(\mathrm{P}<0.05)$, whereas the expression levels of the two factors indicated no significant difference between the suture-induced and control groups (Fig. 5). Thus, these results demonstrate that the CXCL12/CXCR4 signaling pathway is dependent on the VEGF-C/VEGFR-3 pathway.

\section{Discussion}

To the best of our knowledge, the present study is the first to demonstrate that CXCL12/CXCR4 regulates $\mathrm{LG}$ in vivo in a corneal suture-induced mouse model. Giacomini et al (34) quantified and compared HA and LG following alkali burnand suture-induced CNV. They demonstrated that LG was more marked in the suture-induced model as compared with the alkali burn model, and the two types induced similar HA. Thus, the present study used a suture-induced CNV mouse model.

In the current study, there are low levels of expression of CXCL12/CXCR4 in normal cornea analyzed by RT-qPCR and immunohistochemistry. Immunohistochemistry results demonstrated that CXCR4 was present in normal corneal epithelium. Bourcier et al (35) detected CXCL12/CXCR4 mRNA expression levels in normal human corneal by in situ hybridization with similar results to those in the present study. CXCL12/CXCR4 expression levels in suture-induced CNV was investigated by immunohistochemical analysis, RT-qPCR and western blotting. The present study observed SDF-1 and CXCR4 mRNA overexpression on day 7 in suture-induced CNV, consistent with a previous study (36). These findings suggest that suture-induced inflammation increases CXCL12/CXCR4 expression levels in the cornea, and that CXCL12/CXCR4 is crucial to the induction of inflammatory corneal HA and LG. AMD3100 is a specific antagonist of CXCR4, with no cross reaction with other chemokines. According to the immunofluorescence results, corneal HA and LG were markedly reduced in eyes receiving subconjunctival injections of AMD3100 compared with the suture placement group. Subconjunctival injection of AMD3100 was an effective method to decrease the expression of CXCL12/CXCR4. These results suggest that CXCL12/CXCR4 was involved in CNV and, particularly, LG.

Cornea avascularity is maintained by the balance between angiogenic and anti-angiogenic factor expression $(37,38)$. Corneal wounding enhances predominantly the expression of angiogenic factors, including VEGF and bFGF $(25,39)$, and skewed the balance toward angiogenesis, thus resulting in CNV. The crucial role of VEGF-A in the pathophysiology of CNV has been extensively previously reported $(15,40,41)$. CXCL12 and its receptor CXCR4 promote glioma stem cell-initiated glioma growth and angiogenesis by stimulating VEGF-A production (25). Antoniou et al (42) demonstrated VEGF-A and SDF-1 
mRNA coexpression in angiogenesis as part of the pathogenesis of idiopathic pulmonary fibrosis. Mirshahi et al (27) demonstrated that SDF-1 induces an angiogenic effect in vitro and in rabbit corneal pocket. SDF-1 was associated with a slight increase in VEGF production (42). In the present study, VEGF-A/VEGFR-1 mRNA expression levels were upregulated in the suture-induced and control groups, whereas VEGF-A/VEGFR-1 expression levels were downregulated in corneas treated with AMD3100. The results are consistent with the results from investigation of CXCL12/CXCR4, thus, the current study hypothesizes CXCL12/CXCR4 may enhance CNV by increasing VEGFA/VEGFR-1.

Among the known lymphangiogenic factors, VEGF-C, which exerts its functions via VEGFR-3, is the most potent and specific growth factor acting directly on lymphatic vessels. Although bFGF, angiopoient-1/2, insulin-like growth factor-1/2, hepatocyte growth factor, and PDGF-BB have been demonstrated to be prolymphangiogenesis factors, the VEGF-C/VEGFR-3 signaling pathway is common for a number of lymphangiogenic factors. bFGF has also been reported to upregulate VEGF-C expression in endothelial cells, and its lymphangiogenic property is mediated by VEGF-C (43). The present study hypothesized that CXCL12/CXCR4 activates a signaling pathway dependent on that triggered by the VEGF-C/VEGFR-3 axis. The present study has demonstrated that VEGF-C and VEGFR-3 expression were significantly upregulated in the suture placement and control groups, whereas the expression levels of the two factors were downregulated in mice treated with AMD3100. Although it is possible that CXCL12 may also have indirect effects in promoting LG via inducing other factors, the VEGF-C/VEGFR-3 pathway and CXCL12/CXCR4 signaling pathways are involved in LG, and the CXCL12/CXCR4 pathway may depend on VEGF-C/VEGFR-3 pathway in regulating LG. The present study suggests that the CXCL12/CXCR4 axis is a potent positive-regulator of LG.

In conclusion, the results from the present study demonstrate CXCL12/ CXCR4 regulates HA and LG following corneal suture placement, and provides a novel strategy to inhibit LG. Notably, the present study is the first to demonstrate evidence that CXCL12/CXCR4 modulates LG in a corneal suture-induced mouse model.

\section{Acknowledgements}

The present study was supported by grants from the National Natural Science Foundation of China (no. 81470618), the Scientific Research Fund of Heilongjiang Provincial Education Department (no. 12521262) and the Scientific Research Fund of Heilongjiang Provincial Health Bureau (no. 2011-031).

\section{References}

1. Qazi Y, Wong G, Monson B, Stringham J and Ambati BK: Corneal transparency: Genesis, maintenance and dysfunction. Brain Res Bull 81: 198-210, 2010.

2. Chung ES, Chauhan SK, Jin Y, Nakao S, Hafezi-Moghadam A, van Rooijen N, Zhang Q, Chen La and Dana R: Contribution of macrophages to angiogenesis induced by vascular endothelial growth factor receptor-3-specific ligands. Am J Pathol 175: 1984-1992, 2009.
3. Ellenberg D, Azar DT, Hallak JA, Tobaigy F, Han KY, Jain S, Zhou $\mathrm{Z}$ and Chang JH: Novel aspects of corneal angiogenic and lymphangiogenic privilege. Prog Retin Eye Res 29: 208-248, 2010.

4. Hajrasouliha AR, Sadrai Z, Chauhan SK and Dana R: b-FGF induces corneal blood and lymphatic growth in a spatially distinct pattern. Cornea 31: 804-809, 2012.

5. Ebrahem Q, Minamoto A, Hoppe G, Anand-Apte B, Sears E: Triamcinolone acetonide inhibits IL-6- and VEGF-induced angiogenesis downstream of the IL-6 and VEGF receptors. Invest Ophthalmol Vis Sci 47: 4935-4941, 2006.

6. Zhang J, Cao R, Zhang Y, Jia T, Cao Y and Wahlberg E: Differential roles of PDGFR-alpha and PDGFR-beta in angiogenesis and vessel stability. FASEB J 23: 153-163, 2009.

7. Xin X, Yang S, Ingle G, Zlot C, Rangell L, Kowalski J, Schwall R, Ferrara N and Gerritsen ME: Hepatocyte growth factor enhances vascular endothelial growth factor-induced angiogenesis in vitro and in vivo. Am J Pathol 158: 1111-1120, 2001.

8. Kaipainen A, Korhonen J, Mustonen T, van Hinsbergh VW, Fang GH, Dumont D, Breitman M and Alitalo K Expression of the fms-like tyrosine kinase 4 gene becomes restricted to lymphatic endothelium during development. Proc Natl Acad Sci USA 92: 3566-3570, 1995.

9. Banerji S, Ni J, Wang SX, Clasper S, Su J, Tammi R, Jones M and Jackson DG: LYVE-1, a new homologue of the CD44 glycoprotein, is a lymph-specific receptor for hyaluronan. J Cell Biol 144: 789-801, 1999.

10. Wilting J, Papoutsi M, Christ B, Nicolaides $\mathrm{KH}$, von Kaisenberg CS, Borges J, Stark GB, Alitalo K, Tomarev SI, Niemeyer C and Rössler J: The transcription factor Prox 1 is a marker for lymphatic endothelial cells in normal and diseased human tissues. FASEB J 16: 1271-1273, 2002.

11. Tammela $\mathrm{T}$ and Alitalo K. Lymphangiogenesis: Molecular mechanisms and future promise. Cell 140: 460-476, 2010.

12. Jeltsch M, Kaipainen A, Joukov V, Meng X, Lakso M, Rauvala H, Swartz M, Fukumura D, Jain RK and Alitalo K: Hyperplasia of lymphatic vessels in VEGF-C transgenic mice. Science 276: 1423-1425, 1997.

13. Karkkainen MJ, Haiko P, Sainio K, Partanen J, Taipale J, Petrova TV, Jeltsch M, Jackson DG, Talikka M, Rauvala H, et al: Vascular endothelial growth factor $\mathrm{C}$ is required for sprouting of the first lymphatic vessels from embryonic veins. Nat Immunol 5: 74-80, 2004.

14. Mäkinen T, Jussila L, Veikkola T, Karpanen T, Kettunen MI, Pulkkanen KJ, Kauppinen R, Jackson DG, Kubo H, Nishikawa $\mathrm{S}$, et al: Inhibition of lymphangiogenesis with resulting lymphedema in transgenic mice expressing soluble VEGF receptor-3. Nat Med 7: 199-205, 2001.

15. Amano S, Rohan R, Kuroki M, Tolentino M and Adamis AP: Requirement for vascular endothelial growth factor in woundand inflammation-related corneal neovascularization. Invest Ophthalmol Vis Sci 39: 18-22, 1998.

16. Murphy PM, Baggiolini M, Charo IF, Hébert CA, Horuk R, Matsushima K, Miller LH, Oppenheim JJ and Power CA: International union of pharmacology. XXII. Nomenclature for chemokine receptors. Pharmacol Rev 52: 145-176, 2000.

17. Nagasawa T, Hirota S, Tachibana K, et al: Defects of B-cell lymphopoiesis and bone-marrow myelopoiesis in mice lacking the CXC chemokine PBSF/SDF-1. Nature 382: 635-638, 1996.

18. Zou YR, Kottmann AH, Kuroda M, Taniuchi I and Littman DR. Function of the chemokine receptor CXCR4 in haematopoiesis and in cerebellar development. Nature 393: 595-599, 1998.

19. Ma Q, Jones D, Borghesani PR, et al: Impaired B-lymphopoiesis, myelopoiesis, and derailed cerebellar neuron migration in CXCR4- and SDF-1-deficient mice. Proc Natl Acad Sci USA 95: 9448-53, 1998.

20. Tachibana K, Hirota S, Iizasa H, Yoshida H, Kawabata K, Kataoka Y, Kitamura Y, Matsushima K, Yoshida N, Nishikawa S, et al: The chemokine receptor CXCR4 is essential for vascularization of the gastrointestinal tract. Nature 393: 591-594, 1998.

21. Müller A, Homey B, Soto H, Ge N, Catron D, Buchanan ME, McClanahan T, Murphy E, Yuan W, Wagner SN, et al: Involvement of chemokine receptors in breast cancer metastasis. Nature 410: 50-56, 2001.

22. Burger JA and Kipps TJ: CXCR4: A key receptor in the crosstalk between tumor cells and their microenvironment. Blood 107: 1761-1767, 2006. 
23. Chiang AC and Massagué J: Molecular basis of metastasis. N Engl J Med 359: 2814-2823, 2008.

24. De Clercq E: The bicyclam AMD3100 story. Nat Rev Drug Discov 2: 581-587, 2003

25. Salcedo R, Wasserman K, Young HA, et al: Vascular endothelial growth factor and basic fibroblast growth factor induce expression of CXCR4 on human endothelial cells: In vivo neovascularization induced by stromal-derived factor-1alpha. Am J Pathol 154 $1125-1135,1999$

26. Ping YF, Yao XH, Jiang JY, et al: The chemokine CXCL12 and its receptor CXCR4 promote glioma stem cell-mediated VEGF production and tumour angiogenesis via PI3K/AKT signalling. J Pathol 224: 344-354, 2011

27. Mirshahi F, Pourtau J, Li H, Muraine M, Trochon V, Legrand E, Vannier J, Soria J, Vasse M and Soria C: SDF-1 activity on microvascular endothelial cells: Consequences on angiogenesis in in vitro and in vivo models. Thromb Res 99: 587-594, 2000.

28. Cursiefen C, Chen L, Borges LP, Jackson D, Cao J, Radziejewski C, D'Amore PA, Dana MR, Wiegand SJ and Streilein JW: VEGF-A stimulates lymphangiogenesis and hemangiogenesis in inflammatory neovascularization via macrophage recruitment. J Clin Invest 113: 1040-1050, 2004.

29. Zhang Z, Ma JX, Gao G, Li C, Luo L, Zhang M, Yang W, Jiang A, Kuang W, Xu L, et al: Plasminogen kringle 5 inhibits alkali-burn-induced corneal neovascularization. Invest Ophthalmol Vis Sci 46: 4062-4071, 2005.

30. Livak KJ and Schmittgen TD: Analysis of relative gene expression data using real-time quantitative PCR and the 2(-Delta Delta C(T)) Method. Methods 25: 402-408, 2001

31. Tang XL, Sun JF, Wang XY, Du LL and Liu P: Blocking neuropilin-2 enhances corneal allograft survival by selectively inhibiting lymphangiogenesis on vascularized beds. Mol Vis 16 : 2354-2361, 2010

32. Bock F, Onderka J, Hos D, Horn F, Martus P and Cursiefen C: Improved semiautomatic method for morphometry of angiogenesis and lymphangiogenesis in corneal flatmounts. Exp Eye Res 87: 462-470, 2008.

33. Cao R, Björndahl MA, Religa P, et al: PDGF-BB induces intratumoral lymphangiogenesis and promotes lymphatic metastasis. Cancer Cell 6: 333-345, 2004.
34. Giacomini C, Ferrari G, Bignami F and Rama P: Alkali burn versus suture-induced corneal neovascularization in C57BL/6 mice: An overview of two common animal models of corneal neovascularization. Exp Eye Res 121: 1-4, 2014.

35. Bourcier T, Berbar T, Paquet S, Rondeau N, Thomas F, Borderie V, Laroche L, Rostène W, Haour F and Lombet A: Characterization and functionality of CXCR4 chemokine receptor and SDF-1 in human corneal fibroblasts. Mol Vis 9: 96-102, 2003.

36. Carr AN, Howard BW, Yang HT, Eby-Wilkens E, Loos P, Varbanov A, Qu A, DeMuth JP, Davis MG and Proia A: Efficacy of systemic administration of SDF-1 in a model of vascular insufficiency: Support for an endothelium-dependent mechanism. Cardiovasc Res 69: 925-935, 2006.

37. Cursiefen C, Masli S, Ng TF, Dana MR, Bornstein P, Lawler J and Streilein JW: Roles of thrombospondin-1 and -2 in regulating corneal and iris angiogenesis. Invest Ophthalmol Vis Sci 45: 1117-1124, 2004.

38. Gao G and Ma J: Tipping the balance for angiogenic disorders. Drug Discov Today 7: 171-172, 2002.

39. Uno K, Hayashi H, Kuroki M, Uchida H, Yamauchi Y, Kuroki M and Oshima K: Thrombospondin-1 accelerates wound healing of corneal epithelia. Biochem Biophys Res Commun 315: 928-934, 2004.

40. Bock F, Onderka J, Dietrich T, Bachmann B, Kruse FE, Paschke M, Zahn G and Cursiefen C: Bevacizumab as a potent inhibitor of inflammatory corneal angiogenesis and lymphangiogenesis. Invest Ophthalmol Vis Sci 48: 2545-2552, 2007.

41. Manzano RP, Peyman GA, Khan P, Carvounis PE, Kivilcim M, Ren M, Lake JC and Chévez-Barrios P: Inhibition of experimental corneal neovascularisation by bevacizumab (Avastin). Br J Ophthalmol 91: 804-807, 2007.

42. Antoniou KM, Soufla G, Lymbouridou R, Economidou F, Lasithiotaki I, Manousakis M, Drositis I, Spandidos DA and Siafakas NM: Expression analysis of angiogenic growth factors and biological axis CXCL12/CXCR4 axis in idiopathic pulmonary fibrosis. Connect Tissue Res 51: 71-80, 2010.

43. Chang LK, Garcia-Cardeña G, Farnebo F, Fannon M, Chen EJ, Butterfield C, Moses MA, Mulligan RC, Folkman J and Kaipainen A: Dose-dependent response of FGF-2 for lymphangiogenesis. Proc Natl Acad Sci USA 101: 11658-11663, 2004. 